EUROPEAN JOURNAL OF PURE AND APPLIED MATHEMATICS

Vol. 13, No. 1, 2020, 144-157

ISSN 1307-5543 - www.ejpam.com

Published by New York Business Global

\title{
Numerical methods for advection problem
}

\author{
Diogène Vianney Pongui Ngoma ${ }^{1}$, Germain Nguimbi ${ }^{1 *}$, Vital Delmas Mabonzo ${ }^{2}$, \\ Narcisse Batangouna ${ }^{1}$ \\ 1 Ecole Nationale Supérieure Polytechnique, Marien Ngouabi University, Congo \\ 2 Parcours Mathématiques, E.N.S, Marien Ngouabi University, Brazzaville, Congo
}

\begin{abstract}
This paper aims is to solve an advection problem where $u=u(x, t)$ is the solution by Lax-Wendrof and finite difference methods, to study the analytical stability in $L^{2}[0,1], L^{\infty}[0,1]$, then calculate the truncation error of these methods and finally study the analytical convergence of these methods. These numerical techniques of resolution were implemented in Scilab.
\end{abstract}

2020 Mathematics Subject Classifications: 65M06, 65M12, 65K05, 65L12

Key Words and Phrases: Advection problem, truncation error, stability, convergence, LaxWendroff and finite difference methods

\section{Introduction}

Many disciplines of physics consist of describing phenomena of transport, heat and induction. To describe such phenomena, it seems quite natural to describe the evolution of certain physical quantities in time as well as in space. Since they involve several parameters, the differential equations involve partial derivatives with respect to each parameter. Hence the term "partial differential equations" in short PDEs. The PDEs are also involved in the mathematical study of many problems encountered in various fields of science (mechanics, chemistry, economics, biology, etc.), as well as in various applied fields, or even advanced industrial, mainly in engineering and oil industry.

A PDE in itself does not have a pure solution, because in general it is difficult to find a solution $u$ to it in a unique way with no limit condition [1-3, 5, 8, 11]. After modeling a physical problem (a visible problem) we get an invisible problem (a mathematical equation: partial differential equations for example), but PDEs are usually very complex to solve, or they have solutions for particular cases, but also the random phenomena of nature lead to nonlinear equations which gives a complexity to the mathematical model studied.

The principle of solving partial differential equations is to replace a complex system into a simple object or operator by leaving the main aspects of the original, which is called

${ }^{*}$ Corresponding author.

DOI: https://doi.org/10.29020/nybg.ejpam.v13i1.3619

Email addresses: diogene.ponguingoma@umng.cg (D.V. Pongui Ngoma), germain.nguimbi@umng.cg (G.Nguimbi), vitalm28@gmail.com (V. D. Mabonzo) 
a numerical resolution. We want to solve the advection problem of order one in time and space (1) of solution $u=u(x, t)$ by finite difference and Lax-Wendroff methods $[4-6,9,10]$.

$$
\frac{\partial u}{\partial t}+\alpha \frac{\partial u}{\partial x}=0
$$

where $\alpha$ is the advection coefficient, subject to the homogeneous Dirichlet conditions

$$
u(0, t)=u(1, t)=0,
$$

going from the initial solution

$$
u(x, 0)=u_{0}(x)=\sin (19 \pi x) .
$$

These methods will have to calculate the solution $u$ of the problem for different steps in space and time in order to represent on the same graph the numerical solutions of the finite difference and Lax-Wendroff methods for the equation (1), we will study the analytical stability in $L^{2}([0,1])$ and $L^{\infty}([0,1])$ of finite difference and Lax-Wendroff methods for the advection equation, then we will calculate the truncation error of these methods. We will study the analytical convergence of each of these methods.

All these numerical methods will be implemented with the Scilab software.

\section{Advection problem}

The advection or linear transport equation is a hyperbolic PDE, it is the simplest one and consists in finding the solution $u=u(x, t) \in \mathbb{R}$ such that [8]

$$
\frac{\partial u}{\partial t}+\alpha \frac{\partial u}{\partial x}=0
$$

where $\alpha>0$ is the advection coefficient.

For the purpose of the resolution we will consider the homogeneous Dirichlet boundary conditions

$$
u(0, t)=u(1, t)=0 .
$$

And the solution is initiated from $u(x, 0)=u_{0}(x)=\sin (19 \pi x)$.

\section{Numerical resolution of advection problem}

In this section, we will calculate the solution of the advection model by using two numerical methods including the finite difference method and that of Lax-Wendroff.

\subsection{Resolution by the finite difference method}

We shall now calculate the solution $u(x, t)$ of the advection problem for different steps in space and time. For this purpose, the finite difference scheme of the advection problem is written:

$$
\frac{u_{j}^{n+1}-u_{j}^{n}}{\Delta t}+\alpha \frac{u_{j}^{n}-u_{j-1}^{n}}{\Delta x}=0, \quad j \in \mathbb{Z}, n \geqslant 0,
$$


D.V. Pongui Ngoma, G.Nguimbi, V.D.Mabonzo, N. Batangouna / Eur. J. Pure Appl. Math, 13 (1) (2020), 144-157 146 and in the form of recurrence

$$
u_{j}^{n+1}=\beta u_{j-1}^{n}+(1-\beta) u_{j}^{n}, \quad \text { with } \quad \beta=\alpha \frac{\Delta t}{\Delta x}
$$

The homogeneous Dirichlet boundary conditions are

$$
\begin{gathered}
u(0, t) \simeq u\left(x_{0}, t_{n}\right)=u_{0}^{n}=0, \\
u(1, t) \simeq u\left(x_{N+1}, t_{n}\right)=u_{N+1}^{n}=0,
\end{gathered}
$$

By varying the index $j=1,2,3, \ldots, N$ of the equation (4), we obtain:

$$
\left(\begin{array}{c}
u_{1}^{n+1} \\
u_{2}^{n+1} \\
u_{3}^{n+1} \\
\vdots \\
u_{N}^{n+1}
\end{array}\right)=\left(\begin{array}{cccccc}
1-\beta & 0 & & 0 & \ldots & 0 \\
\beta & 1-\beta & 0 & \ldots & 0 \\
0 & \beta & & 1-\beta & \ldots & 0 \\
\vdots & & \ddots & & \ddots & \vdots \\
0 & 0 & \ldots & \beta & & 1-\beta
\end{array}\right)\left(\begin{array}{c}
u_{1}^{n} \\
u_{2}^{n} \\
u_{3}^{n} \\
\vdots \\
u_{N}^{n}
\end{array}\right)
$$

\subsection{Resolution by Lax-Wendroff method}

We will calculate the solution $u(x, t)$ of the advection problem for different steps in space and time. For this purpose, the Lax-Wendroff scheme for the advection equation is written as follows

$$
\frac{u_{j}^{n+1}-u_{j}^{n}}{\Delta t}+c \frac{u_{j+1}^{n}-u_{j-1}^{n}}{2 \Delta x}-\left(\frac{c^{2} \Delta t}{2}\right) \frac{u_{j-1}^{n}-2 u_{j}^{n}+u_{j+1}^{n}}{\Delta x^{2}}=0
$$

and in the form of recurrence

$$
u_{j}^{n+1}=\left(1-\lambda^{2}\right) u_{j}^{n}+\left(\frac{\lambda^{2}}{2}+\frac{\lambda}{2}\right) u_{j-1}^{n}+\left(\frac{\lambda^{2}}{2}-\frac{\lambda}{2}\right) u_{j+1}^{n}, \quad \text { avec } \quad \lambda=c \frac{\Delta t}{\Delta x} .
$$

The homogeneous Dirichlet boundary conditions are

$$
\begin{gathered}
u(0, t) \simeq u\left(x_{0}, t_{n}\right)=u_{0}^{n}=0, \\
u(1, t) \simeq u\left(x_{N+1}, t_{n}\right)=u_{N+1}^{n}=0,
\end{gathered}
$$

by varying the index $j=1,2,3, \ldots, N$ of the equation (7) we obtain the following matrix system 


$$
\left(\begin{array}{c}
u_{1}^{n+1} \\
u_{2}^{n+1} \\
u_{3}^{n+1} \\
\vdots \\
u_{N}^{n+1}
\end{array}\right)=\left(\begin{array}{cccccc}
\left(1-\lambda^{2}\right) & \left(\frac{\lambda^{2}}{2}-\frac{\lambda}{2}\right) & 0 & 0 & \cdots & 0 \\
\left(\frac{\lambda^{2}}{2}+\frac{\lambda}{2}\right) & \left(1-\lambda^{2}\right) & \left(\frac{\lambda^{2}}{2}-\frac{\lambda}{2}\right) & 0 & \cdots & 0 \\
0 & \left(\frac{\lambda^{2}}{2}+\frac{\lambda}{2}\right) & \left(1-\lambda^{2}\right) & \left(\frac{\lambda^{2}}{2}-\frac{\lambda}{2}\right) \cdots 0 \\
\vdots & \ddots & \ddots & \left(\frac{\lambda^{2}}{2}-\frac{\lambda}{2}\right) \\
0 & \cdots & 0 & \left(\frac{\lambda^{2}}{2}+\frac{\lambda}{2}\right) & \left(1-\lambda^{2}\right)
\end{array}\right)\left(\begin{array}{c}
u_{1}^{n} \\
u_{2}^{n} \\
u_{3}^{n} \\
\vdots \\
u_{N}^{n}
\end{array}\right)
$$

Solving the advection problem by the finite difference and Lax-Wendroff methods, we find that the linear system obtained by the finite difference method admits a bidiagonal and non-symmetric while that obtained by Lax-Wendroff is tridiagonal and non-symmetric.

\subsection{Numerical simulation}

The aim here is to represent on the same graph the solutions of the linear systems (7) and (8) of the finite difference and Lax-Wendroff methods respectively, by making the choice by numerical tests of the parameters: advection velocity $\alpha$, number of steps in space $N$, number of steps in time $T$ and time steps $\Delta t$, respectively. This simulation will be implemented in Scilab. 


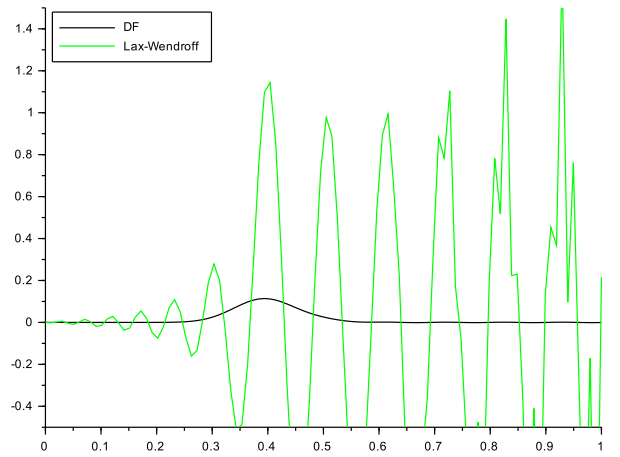

(a)

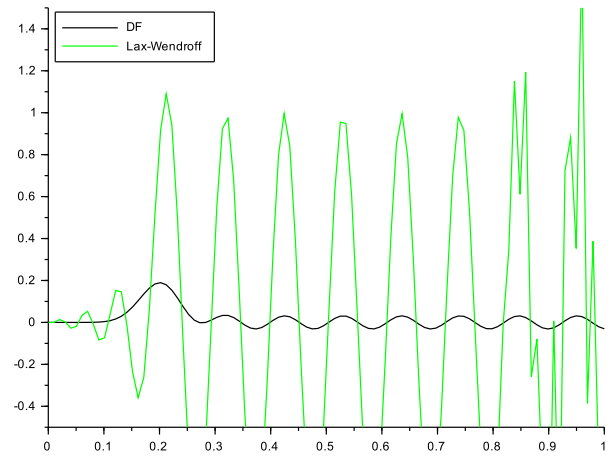

(b)

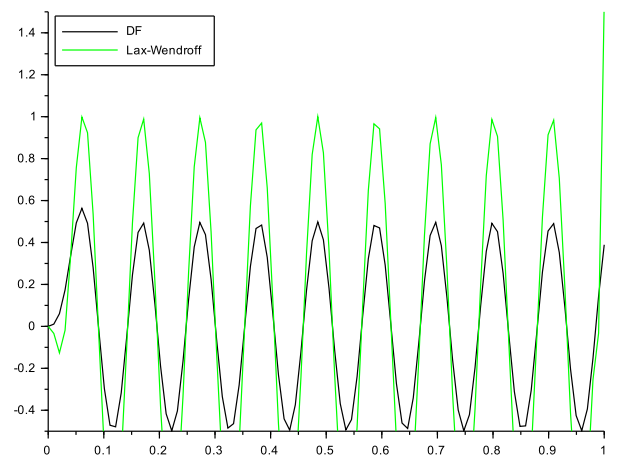

(c)

Figure 1: Representation of the solution of the advection problem by finite difference (FD) and Lax-Wendroff methods for $N=99$ et $T=2000$.

Taking the number of steps in space $N=99$ and the number of steps in time $T=2000$ in finite difference methods (7) and Lax-Wendroff (8), we sought to vary the advection speed $\alpha$ and the time step $\Delta t$ of the advection problem and numerical methods (finite differences and Lax-Wendroff) respectively, in order to verify the numerical convergence of the solutions (7) and Lax-Wendroff (8). ( See figure 1). 
- Taking $\alpha=0.2$ et $\Delta t=0.001$, we note that the solution obtained by the finite difference (FD) method remains constant whereas that obtained by the Lax-Wendroff method is unstable at first, then becomes stable and becomes unstable after (Figure 1 a).

- Taking $\alpha=0.002$ et $\Delta t=0.05$, we find that the solution by FD is constant at the beginning, then oscillates at very low amplitudes whereas by Lax-Wendroff it is unstable at first, then remains stable for a long time and becomes unstable after ( Figure $1 \mathrm{~b}$ ).

- Taking $\alpha=0.02$ et $\Delta t=0.001$, we note that both solutions (FD and LaxWendroff) converge and the solution by Lax-Wendroff admits a higher peak than that obtained by FD ( Figure $1 \mathrm{c}$ ).

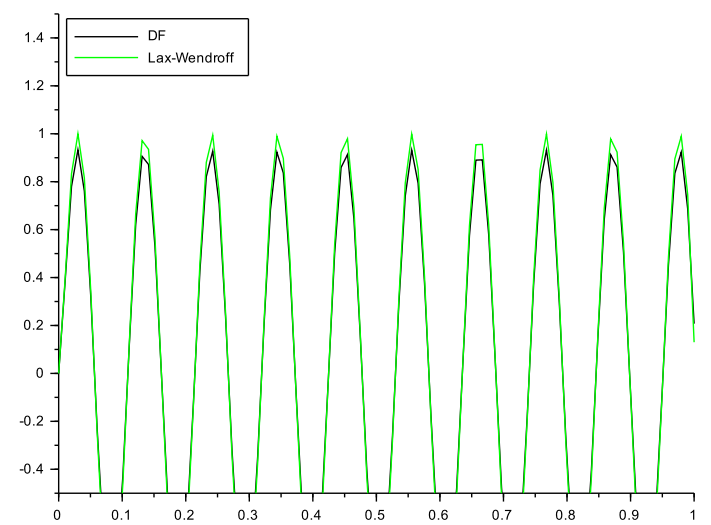

(d)

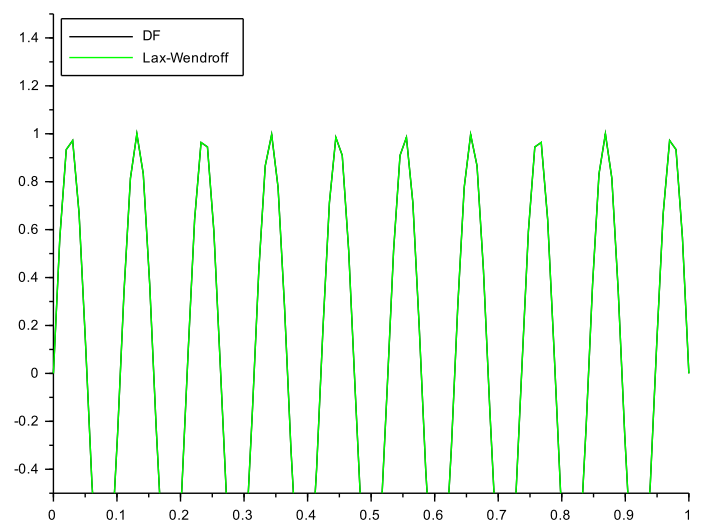

(e)

Figure 2: Representation of the solution of the advection problem by finite difference (FD) and Lax-Wendroff methods for $N=99$ et $T=2000$.

- Taking $\alpha=0.002$ et $\Delta t=0.001$, we find that both solutions (FD and LaxWendroff) converge numerically almost everywhere ( Figure $2 \mathrm{~d}$ ).

- taking $\alpha=0.002$ et $\Delta t=0.000001$, we see that both solutions (FD and LaxWendroff) admit a total convergence numerically( Figure 2 e)

The numerical convergence of the solution from Finite Difference and Lax-Wendroff method for resolving the advection equation requires very good choices of the advection speed parameter $\alpha$ and the time step $\Delta t$, as well as a fairly high number of steps in space and time $(N$ and $T)$. 


\section{Study of the analytical stability in $L^{2}([0 ; 1])$ and $L^{\infty}([0 ; 1])$ of the finite} difference and Lax-Wendroff methods for the advection equation

In this section, we study the analytical stability in $L^{2}([0 ; 1])$ and $L^{\infty}([0 ; 1])$ of the finite difference and Lax-Wendroff methods for the advection equation

\subsection{Analytical stability in $L^{2}([0 ; 1])$ for the finite difference method of the transport equation}

Here, we will study the analytical stability in $L^{2}([0 ; 1])$ of the numerical finite difference method used for the advection problem.

Let $e^{i w x}$ and $e^{i w x_{j}}$ be the mode values of the exact and discrete operators respectively where $i$ is the imaginary unit such that $i^{2}=-1$.

Let $u^{n}=u_{w}^{n} e^{i w x}, i \in \mathbb{C}$ and $x_{j}=j \Delta x \simeq j h$ where $h=\Delta x$ is the constant step of space discretization.

Then

$$
u_{j}^{n}=u_{w}^{n} e^{i w x_{j}} \simeq u_{w}^{n} e^{i w j h}
$$

Replacing (9) in (4), we obtain

$$
\begin{gathered}
u_{w}^{n+1} e^{i w j h}=\beta u_{w}^{n} e^{i w j h} \cdot e^{-i w h}+(1-\beta) u_{w}^{n} e^{i w j h}, \\
u_{w}^{n+1}=\left[\beta e^{-i w h}+(1-\beta)\right] u_{w}^{n}, \quad w h \in[-\pi ; \pi]
\end{gathered}
$$

Let us put $\quad r(w h)=\beta e^{-i w h}+(1-\beta)$, where $r(w h)$ is the amplification coefficient of the finite difference operator .

Let us show that $|r(w h)| \leqslant 1$,

$$
\begin{aligned}
r(w h)^{2} & =1-2 \beta+2 \beta \cos w h-2 \beta^{2} \cos w h+\beta^{2}+\beta^{2}, \\
& =(1-\beta)^{2}+\beta^{2}+2 \beta \cos w h(1-\beta), \\
& =(1-\beta)^{2}+2 \beta(1-\beta) \cos w h+\beta^{2}, \\
|r(w h)|^{2} & =\left|(1-\beta)^{2}+2 \beta(1-\beta) \cos w h\right|+\beta^{2}, \\
|r(w h)|^{2} & \leqslant(1-\beta)^{2}+2 \beta|(1-\beta)|+\beta^{2}=[(1-\beta)+\beta]^{2} \leqslant 1,
\end{aligned}
$$

where

$$
|r(w h)| \leqslant 1 .
$$

This reflects the analytical stability in $L^{2}([0 ; 1])$ of the finite difference method for the transport equation. 
D.V. Pongui Ngoma, G.Nguimbi, V.D.Mabonzo, N. Batangouna / Eur. J. Pure Appl. Math, 13 (1) (2020), 144-157 151

\subsection{Analytical stability in $L^{2}([0 ; 1])$ for the Lax-Wendroff method of the transport equation}

We shall want to study the analytical stability in $L^{2}([0 ; 1])$ of the Lax-Wendroff method used for the advection problem. Thus let us use the Von-Neuman condition.

The Lax-Wendroff scheme for the advection equation is

$$
u_{j}^{n+1}=u_{j}^{n}-\lambda \frac{u_{j+1}^{n}-u_{j-1}^{n}}{2}+\lambda^{2} \frac{u_{j-1}^{n}-2 u_{j}^{n}+u_{j+1}^{n}}{2} .
$$

According to the Von-Neumann stability criterion:

$$
\begin{gathered}
A=\frac{u^{n+1}}{u^{n}} \\
\frac{u^{n+1}}{u^{n}}=\frac{u_{j}^{n}-\frac{\lambda}{2}\left(u_{j+1}^{n}-u_{j-1}^{n}\right)+\frac{\lambda^{2}}{2}\left(u_{j-1}^{n}-2 u_{j}^{n}+u_{j+1}^{n}\right)}{u^{n}}, \\
=\frac{\left(\frac{\lambda^{2}}{2}+\frac{\lambda}{2}\right) u_{j-1}^{n}+\left(1-\lambda^{2}\right) u_{j}^{n}+\left(\frac{\lambda^{2}}{2}-\frac{\lambda}{2}\right) u_{j+1}^{n}}{u^{n}} .
\end{gathered}
$$

Let us take two mode values of the exact and discrete operators $e^{i k \Delta x}$ and $e^{i k j \Delta x}$ respectively.

Let be

$$
u_{j}^{n}=e^{i k j \Delta x} u_{k}^{n}
$$

Equation (12) then becomes

$$
\begin{aligned}
A & =\frac{\left(\frac{\lambda^{2}}{2}+\frac{\lambda}{2}\right) u_{k}^{n} \cdot e^{i k(j-1) \Delta x}+\left(1-\lambda^{2}\right) u_{k}^{n} \cdot e^{i k j \Delta x}+\left(\frac{\lambda^{2}}{2}-\frac{\lambda}{2}\right) u_{k}^{n} \cdot e^{i k(j+1) \Delta x}}{u_{k}^{n} \cdot e^{i k j \Delta x}}, \\
& =\frac{\left(\frac{\lambda^{2}}{2}+\frac{\lambda}{2}\right) u_{k}^{n} \cdot e^{i k j \Delta x} e^{-i k \Delta x}+\left(1-\lambda^{2}\right) u_{k}^{n} \cdot e^{i k j \Delta x}+\left(\frac{\lambda^{2}}{2}-\frac{\lambda}{2}\right) u_{k}^{n} \cdot e^{i k j \Delta x} e^{i k \Delta x}}{u_{k}^{n} \cdot e^{i k j \Delta x}}, \\
& =\frac{\left[\left(\frac{\lambda^{2}}{2}+\frac{\lambda}{2}\right) e^{-i k \Delta x}+\left(1-\lambda^{2}\right)+\left(\frac{\lambda^{2}}{2}-\frac{\lambda}{2}\right) e^{i k \Delta x}\right] u_{k}^{n} \cdot e^{i k j \Delta x}}{u_{k}^{n} \cdot e^{i k j \Delta x}}
\end{aligned}
$$

After simplification, we obtain

$$
\begin{aligned}
& A=\lambda^{2} \cos (k \Delta x)-i \lambda \sin (k \Delta x)+1-\lambda^{2}, \\
& A=1-\lambda^{2}[1-\cos (k \Delta x)]-i \lambda \sin (k \Delta x) .
\end{aligned}
$$

By taking the semi-norm of $A$

$$
|A|=\left|1-\lambda^{2}[1-\cos (k \Delta x)]-i \lambda \sin (k \Delta x)\right|,
$$


D.V. Pongui Ngoma, G.Nguimbi, V.D.Mabonzo, N. Batangouna / Eur. J. Pure Appl. Math, 13 (1) (2020), $144-157$ 152

$$
\begin{gathered}
|A|^{2}=\left|1-\lambda^{2}[1-\cos (k \Delta x)]\right|^{2}+\lambda^{2} \sin ^{2}(k \Delta x), \\
|A| \leqslant 1
\end{gathered}
$$

because

$$
\begin{gathered}
1-\lambda^{2} \geqslant 0, \\
\lambda^{2} \leqslant 1 .
\end{gathered}
$$

Thus, the scheme is stable in the $L^{2}([0,1])$ norm for the Lax-Wendroff method of the CFL transport equation $\lambda \leqslant 1 \quad[4,8]$.

\subsection{Analytical stability in $L^{\infty}([0 ; 1])$ for the finite difference method of the transport equation}

We may now study the analytical stability in $L^{\infty}([0 ; 1])$ of the finite difference method used for the advection problem.

Let us then use, the maximum principe.

Considering the linear interpolation between $u_{j-1}^{n}$ and $u_{j}^{n}$ of the equation (4), we then get

$$
u_{j}^{n+1}=\beta u_{j-1}^{n}+(1-\beta) u_{j}^{n} \leqslant \max _{j \in \mathbb{Z}}\left(u_{j-1}^{n}, u_{j}^{n}\right)
$$

Therefore :

$$
u_{j}^{n+1} \leqslant \max _{j \in \mathbb{Z}}\left(u_{j-1}^{n}, u_{j}^{n}\right) .
$$

By passing to semi-norm and supremum, we obtain

$$
\begin{gathered}
\left.\left|u_{j}^{n+1}\right| \leqslant \max _{j \in \mathbb{Z}}\left(\left|u_{j-1}^{n}\right|, \mid u_{j}^{n}\right) \mid\right), \\
\left.\sup \left|u_{j}^{n+1}\right| \leqslant \sup \max _{j \in \mathbb{Z}}\left(\left|u_{j-1}^{n}\right|, \mid u_{j}^{n}\right) \mid\right), \\
\left\|u^{n+1}\right\|_{\infty} \leqslant\left\|u^{n}\right\|_{\infty} .
\end{gathered}
$$

By simple recurrence, we have

$$
\begin{aligned}
& \text { for } n=0, \quad\left\|u^{1}\right\|_{\infty} \leqslant\left\|u^{0}\right\|_{\infty} \\
& \text { for } n=1, \quad\left\|u^{2}\right\|_{\infty} \leqslant\left\|u^{1}\right\|_{\infty} \leqslant\left\|u^{0}\right\|_{\infty} \\
& \text { for } n=2, \quad\left\|u^{3}\right\|_{\infty} \leqslant\left\|u^{2}\right\|_{\infty} \leqslant\left\|u^{0}\right\|_{\infty}
\end{aligned}
$$$$
\text { at } \operatorname{rank} n, \quad\left\|u^{n}\right\|_{\infty} \leqslant\left\|u^{0}\right\|_{\infty}=C
$$

Then

$$
\left.\left\|u^{n}\right\|_{\infty} \leqslant C \text { ( } C \text { constant }\right) .
$$

Which proves the analytical stability in $L^{\infty}([0 ; 1])$ for the finite difference method of the transport equation. 


\subsection{Analytical stability in $L^{\infty}([0 ; 1])$ for the Lax-Wendroff method of the transport equation}

We may now study the analytical stability in $L^{\infty}([0 ; 1])$ of the finite difference method used for the advection problem. we may then use the maximum principe.

Considering the linear interpolation between $u_{j-1}^{n}, u_{j}^{n}$ and $u_{j+1}^{n}$ of the equation (7), we have:

$$
u_{j}^{n+1}=\left(1-\lambda^{2}\right) u_{j}^{n}+\left(\frac{\lambda^{2}}{2}+\frac{\lambda}{2}\right) u_{j-1}^{n}+\left(\frac{\lambda^{2}}{2}-\frac{\lambda}{2}\right) u_{j+1}^{n} \leqslant \max _{j \in \mathbb{Z}}\left(u_{j-1}^{n}, u_{j}^{n}, u_{j+1}^{n}\right),
$$

Therefore

$$
u_{j}^{n+1} \leqslant \max _{j \in \mathbb{Z}}\left(u_{j-1}^{n}, u_{j}^{n}, u_{j+1}^{n}\right) .
$$

By passing to semi-norm and supremum, we obtain

$$
\begin{gathered}
\left|u_{j}^{n+1}\right| \leqslant \max _{j \in \mathbb{Z}}\left(\left|u_{j-1}^{n}\right|,\left|u_{j}^{n}\right|,\left|u_{j+1}^{n}\right|\right), \\
\sup \left|u_{j}^{n+1}\right| \leqslant \sup \max _{j \in \mathbb{Z}}\left(\left|u_{j-1}^{n}\right|,\left|u_{j}^{n}\right|,\left|u_{j+1}^{n}\right|\right), \\
\left\|u^{n+1}\right\|_{\infty} \leqslant\left\|u^{n}\right\|_{\infty} .
\end{gathered}
$$

by simple recurrence, we have

$$
\begin{aligned}
& \text { for } n=0, \quad\left\|u^{1}\right\|_{\infty} \leqslant\left\|u^{0}\right\|_{\infty} \\
& \text { for } n=1, \quad\left\|u^{2}\right\|_{\infty} \leqslant\left\|u^{1}\right\|_{\infty} \leqslant\left\|u^{0}\right\|_{\infty} \\
& \text { for } n=2, \quad\left\|u^{3}\right\|_{\infty} \leqslant\left\|u^{2}\right\|_{\infty} \leqslant\left\|u^{0}\right\|_{\infty} \\
& \vdots
\end{aligned}
$$

Which proves the analytical stability in $L^{\infty}([0 ; 1])$ for the Lax-Wendroff method of the transport equation.

\section{Analytical convergence of numerical methods}

In this section, we will study the analytical convergence of the finite difference and Lax-Wendroff methods for solving the transport equation. For this purpose, we attempt to compute the truncation errors of these methods. 
D.V. Pongui Ngoma, G.Nguimbi, V.D.Mabonzo, N. Batangouna / Eur. J. Pure Appl. Math, 13 (1) (2020), 144-157 154

\subsection{Truncation error for the finite difference method of the transport equation}

Using Taylor's development of order 2 in relation with time to approach $\frac{\partial u}{\partial t}$, we obtain

$$
\frac{u\left(x_{j}, t_{n+1}\right)-u\left(x_{j}, t_{n}\right)}{\Delta t}=\frac{\partial u}{\partial t}\left(x_{j}, t_{n}\right)+\frac{\Delta t}{2 !} \frac{\partial^{2} u}{\partial t^{2}}\left(x_{j}, t_{n}\right)+O\left(\Delta t^{2}\right)
$$

To approach the derivative $\frac{\partial u}{\partial x}$, let us use Taylor's development of order 2 in relation to space. We then obtain

$$
\alpha \frac{u\left(x_{j}, t_{n}\right)-u\left(x_{j-1}, t_{n}\right)}{\Delta x}=\alpha \frac{\partial u}{\partial x}\left(x_{j}, t_{n}\right)-\alpha \frac{\Delta x}{2 !} \frac{\partial^{2} u}{\partial x^{2}}\left(x_{j}, t_{n}\right)+O\left(\Delta x^{2}\right)
$$

We then define the truncation error of the transport equation for the finite difference method by

$$
\zeta_{j}^{n}=\frac{u\left(x_{j}, t_{n+1}\right)-u\left(x_{j}, t_{n}\right)}{\Delta t}+\alpha \frac{u\left(x_{j}, t_{n}\right)-u\left(x_{j-1}, t_{n}\right)}{\Delta x} .
$$

By adding the equations (13) and (14) member to member, we get

$$
\zeta_{j}^{n}=\frac{\partial u}{\partial t}\left(x_{j}, t_{n}\right)+\alpha \frac{\partial u}{\partial x}\left(x_{j}, t_{n}\right)+\frac{\Delta t}{2 !} \frac{\partial^{2} u}{\partial t^{2}}\left(x_{j}, t_{n}\right)-\alpha \frac{\Delta x}{2 !} \frac{\partial^{2} u}{\partial x^{2}}\left(x_{j}, t_{n}\right)+O\left(\Delta t^{2}+\Delta x^{2}\right)
$$

knowing that

as a result

$$
\frac{\partial u}{\partial t}\left(x_{j}, t_{n}\right)+\alpha \frac{\partial u}{\partial x}\left(x_{j}, t_{n}\right)=0
$$

$$
\zeta_{j}^{n}=\frac{\Delta t}{2 !} \frac{\partial^{2} u}{\partial t^{2}}\left(x_{j}, t_{n}\right)-\alpha \frac{\Delta x}{2 !} \frac{\partial^{2} u}{\partial x^{2}}\left(x_{j}, t_{n}\right)+O\left(\Delta t^{2}+\Delta x^{2}\right) .
$$

We obtain a truncation error of the equation of transport of order 2 in space and in time.

Expressing $\frac{\partial^{2} u}{\partial t^{2}}$ in terms of $\frac{\partial^{2} u}{\partial x^{2}}$, the truncation error is written

$$
\begin{gathered}
\zeta_{j}^{n}=\frac{\Delta t}{2 !} \alpha^{2} \frac{\partial^{2} u}{\partial x^{2}}\left(x_{j}, t_{n}\right)-\alpha \frac{\Delta x}{2 !} \frac{\partial^{2} u}{\partial x^{2}}\left(x_{j}, t_{n}\right)+O\left(\Delta t^{2}+\Delta x^{2}\right), \\
\zeta_{j}^{n}=\frac{\alpha}{2}(\alpha \Delta t-\Delta x) \frac{\partial^{2} u}{\partial x^{2}}\left(x_{j}, t_{n}\right) .
\end{gathered}
$$

\section{Study of the consistency}

This scheme is said to be consistent if the truncation error goes to zero when the time discretization step $\Delta t$ and the space discretization step $\Delta x$ tend to zero independently. 
According to our analysis, the truncation error is

$$
\zeta_{j}^{n}=\frac{\alpha}{2}(\alpha \Delta t-\Delta x) \frac{\partial^{2} u}{\partial x^{2}}\left(x_{j}, t_{n}\right) .
$$

Doing

$$
(\Delta x, \Delta t) \longrightarrow(0,0)
$$

Therefore

$$
\zeta_{j}^{n} \longrightarrow(0,0)
$$

Which proves the consistency of the finite difference scheme for the transport equation.

Since the scheme is stable and consistent, it is concluded that the numerical solution of $u_{j}^{n}$ of the finite difference scheme for the transport equation is convergent.

\subsection{Truncation error for the Lax-Wendroff method of the transport equa- tion}

The Lax-Wendroff scheme for the advection equation is written as

$$
\frac{u_{j}^{n+1}-u_{j}^{n}}{\Delta t}+c \frac{u_{j+1}^{n}-u_{j-1}^{n}}{2 \Delta x}-\left(\frac{c^{2} \Delta t}{2}\right) \frac{u_{j-1}^{n}-2 u_{j}^{n}+u_{j+1}^{n}}{\Delta x^{2}}=0 .
$$

The truncation error of the transport equation for the Lax-Wendroff method is defined by

$\zeta_{j}^{\prime n}=\frac{u\left(x_{j}, t_{n+1}\right)-u\left(x_{j}, t_{n}\right)}{\Delta t}+c \frac{u\left(x_{j+1}, t_{n}\right)-u\left(x_{j-1}, t_{n}\right)}{2 \Delta x}-\left(\frac{c^{2} \Delta t}{2}\right) \frac{u\left(x_{j+1}, t_{n}\right)-2 u\left(x_{j}, t_{n}\right)+u\left(x_{j-1}, t_{n}\right)}{\Delta x^{2}}$

Let us make a development of Taylor in $x$ around the point $x_{j}$ and in $t$ around the point $t_{n}$. Since $u$ is the solution of the transport equation (1), we have

$$
\begin{gathered}
\frac{u\left(x_{j}, t_{n+1}\right)-u\left(x_{j}, t_{n}\right)}{\Delta t}=\frac{\partial u}{\partial t}\left(x_{j}, t_{n}\right)+\frac{\Delta t}{2 !} \frac{\partial^{2} u}{\partial t^{2}}\left(x_{j}, t_{n}\right)+O\left(\Delta t^{2}\right), \\
c \frac{u\left(x_{j+1}, t_{n}\right)-u\left(x_{j-1}, t_{n}\right)}{2 \Delta x}=c \frac{\partial u}{\partial x}\left(x_{j}, t_{n}\right) \\
\left(\frac{c^{2} \Delta t}{2}\right) \frac{u_{j-1}^{n}-2 u_{j}^{n}+u_{j+1}^{n}}{\Delta x^{2}}=\left(\frac{c^{2}}{2}\right) \Delta t \frac{\partial^{2} u}{\partial x^{2}}\left(x_{j}, t_{n}\right) .
\end{gathered}
$$

By inserting the relations (20), (21) and (22) into the expression of $\zeta_{j}^{\prime n}$, we get

$$
\zeta_{j}^{\prime n}=\frac{\Delta t}{2 !} \frac{\partial^{2} u}{\partial t^{2}}\left(x_{j}, t_{n}\right)-\left(\frac{c^{2}}{2}\right) \Delta t \frac{\partial^{2} u}{\partial x^{2}}\left(x_{j}, t_{n}\right)+O\left(\Delta t^{2}+\Delta x^{2}\right)
$$


The truncation error for the Lax-Wendroff method of the transport equation can be written

$$
\begin{gathered}
\zeta_{j}^{\prime n}=\frac{\Delta t}{2}\left[\frac{\partial^{2} u}{\partial t^{2}}\left(x_{j}, t_{n}\right)-c^{2} \frac{\partial^{2} u}{\partial x^{2}}\left(x_{j}, t_{n}\right)\right]+O\left(\Delta t^{2}+\Delta x^{2}\right), \\
=\frac{\Delta t}{2}\left[c^{2} \frac{\partial^{2} u}{\partial x^{2}}\left(x_{j}, t_{n}\right)-c^{2} \frac{\partial^{2} u}{\partial x^{2}}\left(x_{j}, t_{n}\right)\right]+O\left(\Delta t^{2}+\Delta x^{2}\right), \\
\zeta_{j}^{\prime n}=O\left(\Delta t^{2}+\Delta x^{2}\right) .
\end{gathered}
$$

We obtain a truncation error depending on $\Delta t$ and $\Delta x$.

\section{Study of the consistency}

Here it is easier to see that the truncation error of the transport equation for the LaxWendroff schem is zero. We can directly deduce the consistency of the schem because it is obvious that $\zeta_{j}^{\prime n} \longrightarrow(0,0)$ when $(\Delta t, \Delta x) \longrightarrow(0,0)$.

Therefore the numerical solution of $u_{j}^{n}$ of the Lax-Wendroff scheme for the transport equation is convergent.

\section{Conclusion}

The work proposed in this paper allowed us not only to highlight the finite difference and Lax-Wendroff methods, but also to discover the importance of these methods in the numerical resolution of the advection problem. In this paper, we performed a numerical resolution of the advection problem using finite difference and Lax-Wendroff methods. Thus, we obtained linear systems whose matrices are bidiagonal non-symmetric (Finite Difference method), non-symmetric tridiagonal for Lax-Wendroff method. Then we proved that the numerical convergence between these solutions is total by taking: $\alpha=0.002, \Delta t=10^{-6}, \quad N=99$ and $T=2000$. In addition, we used the VonNeumann and CFL conditions to prove the analytical stability of the solution $u_{j}^{n}$ of the advection equation from the finite difference and Lax-Wendroff schemes. Finally, we have also proved the analytical convergence of the solution $u_{j}^{n}$ using the truncation error of these methods.

\section{Acknowledgements}

The authors thank the anonym referees of European Journal of Pure and Applied Mathematics, for their valuable comments and suggestions which have led to an improvement of the presentation. 


\section{References}

[1] H. Beghr and G. Harutjunjan, Robin boundary value problem for the Poisson equation, J. Anal. Appl., 4, 29-45, 2006.

[2] Bourchra Bensiali, Guillaume Chiavassa, and Jacques Liandrat. Penalization of Robin Boundary conditions, Applied Numerical Mathematics, 96, 134-152, 2006.

[3] Shao-Gao Deng. Positive solutions for Robin problem involving the p(x)-Laplacian, J. Math. Anal. Appl., 360,548-560, 2009

[4] Marc Ethier and Y. Bourgault.Semi-implicit time-discretization schemes for the bidomaine model. SIAM .J. Numer. Anal. 46: 2443-2468, 2008.

[5] M. Hinze, R. Pinnau, M. Ulbrich, and S. Ulbrich. Mathematical modelling: Theory and Applications. Springer, 2009.

[6] Peter Knabner, and Lutz Angermann. Numerical methods for elliptic and parabolic partial differential equations,(2nd ed.) Springer, 2000.

[7] Loredana Lanzani, and Osvaldo Méndez . The Poisson's problem for the Laplacian with Robin boundary condition in non-smooth domains, Rev. Math. Iberoamericana, 22, 181-204, 2006.

[8] Brigitte Lucquin. Equations aux drives partielles et leurs approximations, Ellipses, 2004.

[9] G. Nguimbi, D. V. Pongui Ngoma, V. D. Mabonzo, B. B. B. Madzou and L. G. Ngoma Bouanga Mathematical and numerical analysis for Neumann boundary value problem of the Poisson equation, Journal of advnces in mathemtics and computer science, 30, $1-13,2019$.

[10] G. Nguimbi, D. V. Pongui Ngoma, V. D. Mabonzo, B. B. B.Madzou and M. J. J. Kokolo On the existence, uniqueness and application of the finite difference method for solving Robin elliptic boundary value problem, Journal of mathematics research, 11, 26-36, 2019.

[11] Aslak Tveito, and Ragnar Winther. Introduction to partial differential equations: a computational approach, Springer-Verlag, 2008. 